\title{
Geometrical Aspects During Formation of Compact Aggregates of Red Blood Cells
}

\author{
A.V.Cardoso*, A.O.Camargos \\ Cetec - Fundação Centro Tecnológico de Minas Gerais, Setor de Análises Químicas \\ Av. José Cândido da Silveira, 2000, 31170-000 Belo Horizonte - MG, Brasil
}

Received: September 27, 2001; Revised: July 10, 2002

\begin{abstract}
In the past forty years considerable progress has been achieved on the knowledge of human blood as a non-Newtonian shear-thinning suspension, whose initial state, that is at rest (stasis) or at very low shear rates, has a gel-like internal structure which is destroyed as shear stress increases. The main goal of this communication is to describe the role of geometrical aspects during RBC (red blood cell) aggregate formation, growth and compaction on naturally aggregate (porcine blood) and non-aggregate (bovine blood) samples. We consider how these aspects coupled with tension equilibrium are decisive to transform red cell linear roleaux to three-dimensional aggregates or clusters. Geometrical aspects are also crucial on the compaction of red blood cell aggregates. These densely packed aggregates could precipitate out of blood- either as dangerous deposits on arterial walls, or as clots which travel in suspension until they block some crucial capillary.
\end{abstract}

Keywords: blood viscosity, RBC aggregation, rheology, hemorheology

\section{Introduction}

Cardiovascular diseases are among the greatest killers of modern times. Aggregates (we define roleaux as linear arrays of red blood cell and aggregates as three-dimensional structures of interconnected RBC roleaux) precipitate out of blood - either as dangerous deposit on arterial walls - or as clots, which travel in suspension until they block some crucial capillary. Nature relies on the rheology of blood to maintain it fluidity and consequently life. As a result the viscous flow characteristics of human blood has caught the attention of scientists for many centuries ${ }^{1}$.

In the past forty years considerable progress has been achieved on the theoretical knowledge of blood as a nonNewtonian shear-thinning liquid ${ }^{2,3}$. The key to this advance is the understanding that blood as a concentrated suspension has a shear-dependent microstructure that is observed at micron $(\mu \mathrm{m})$ size resolution. When at rest or subjected to very low shear rate this structure might exhibit a microscopic three-dimensional network of RBC branching throughout the containing recipient. An interdisciplinary research field associated with this complex rheological con- dition is the phenomenon of erythrocyte aggregation. Red cell aggregation (RCA) play a decisive role in the pathophysiological behavior of blood circulation. Historically this has been recognized since Fahraeus ${ }^{1}$ and it is a subject of increasing scientific interest ${ }^{7}$.

The aggregation of RBC is a reversible phenomenon that occurs with plasma protein bridging the membranes of adjacent hematies. RBC aggregation represents equilibrium between adhesive forces (macromolecules bridging force), repulsive forces due to electrical charges on the cell surface and mechanical forces (shear stress). Adhesive forces is increased in many pathological situations (hypertension, diabetics, thrombosis, etc), in which the shear stress required to break up RBC aggregates, would become elevated in comparison with normal human aggregates.

Differences in red cell aggregation between mammals have been reported ${ }^{8}$ and these differences might help on modelling different levels of human red cell aggregation. Blood aggregation kinetic parameters measured for porcine red cells were close to that of normal human blood ${ }^{4}$.

Although optical or ultrasonic methods provide a prom-

*e-mail: cardoso@cetec.br 
ising way ${ }^{5}$ to study the shear break-up of aggregates during flow there are still many aspects to be solved such as the particle rearrangement induced by hydrodynamic stresses and the influence of the formation of compact aggregates (compaction) on the rheology.

The main goal of this communication is to describe the role of geometrical aspects during RBC aggregates construction on naturally aggregating (porcine blood) and non-aggregating (bovine blood). Further, we hypothesise how geometry is important to enhance the aggregate compaction.

\section{Experimental Procedure}

Whole blood (blood plasma $+\mathrm{RBC}+$ platelets + leukocytes + plasma macromolecules) samples from pigs and cows were collected from local abattoir with the help of SFIA-MA (Brazilian Federal Authority on Animal Health - Ministry of Agriculture) professionals. Blood samples drawn from the vein of healthy animals located at the Experimental Farm/Faculty of Veterinary Medicine of the Federal University of Minas Gerais (UFMG) were also studied and compared with the samples obtained at the abattoir. All blood samples were anticoagulated with EDTA $0.36 \mathrm{~mol} / \mathrm{l}$ in standard tubes for blood collection. To investigate the influence of anticoagulant on the blood rheology the concentration level of EDTA in the blood was set to 1.25, 2.50, 3.75 and $5.0 \% \mathrm{v} / \mathrm{v}$ of anticoagulant. After collection the tubes were immediately transferred to isothermal box where they were kept approximately at $10{ }^{\circ} \mathrm{C}$. On the arrival at the laboratory they were carefully transferred to the freezer and kept at $10{ }^{\circ} \mathrm{C}$ for a maximum period of 7 days. The majority of the viscosity experiments were performed within 4 hours of blood collection. The porcine blood samples were centrifuged (Fanem 206-R) at $1500 \mathrm{~g}$ for $5 \mathrm{~min}$ and the hematocrit of porcine blood was adjusted at $0.40 \mathrm{l} / \mathrm{l}$ by adding or eliminating autologus plasma. The hematocrit of the whole blood was measured with a microcentrifuge (Fanem Centrimicro 211) operating at $10000 \mathrm{~g}$ for $3 \mathrm{~min}$. For observations under optical microscopy the hematocrit on the glass slide was adjusted to low red blood cell volume fraction (hematocrit $\sim 0.10$ ). RBC aggregates micrographs were taken using a Leica microscope equipped with a $35-\mathrm{mm}$ camera.

Blood viscosity was determined with a cone-plate viscometer (Brookfield DVII+) and measurements were taken at the $\left(525-0.1 \mathrm{~s}^{-1}\right)$ shear rate range. Measurements were performed in the temperature interval $38-40{ }^{\circ} \mathrm{C}$ by controlling the temperature with a circulating water equipment (Brookfield TC-500). Prior to blood viscosity measurements the equipment was calibrated with calibrating oils (Cannon Certified Viscosity Standards).

\section{Results and Discussion}

General data information, maximum and minimum viscosity values for porcine, bovine whole blood and plasma measured using a cone-plate type viscometer, are presented in Table 1.Viscosity measurement values at different shear rates for porcine and bovine blood samples, are presented in Fig. 1. The values of porcine blood viscosity (Fig 1a) were taken after the adjustment of the porcine native hematocrit to $40 \%$. Usually, there is a trend of viscosity increase of the native blood as the hematocrit increases ${ }^{6}$. Bovine viscosity data are at native hematocrit. The line between the experimental points is to show the trend followed by blood viscosity of each blood sample as the shear rate is increased at different anticoagulant level. Increasing the concentration of EDTA do not seem to produce a remarkable effect on blood viscosity for both bovine and porcine blood samples. At low shear rate $\left(\sim 10 \mathrm{~s}^{-1}\right)$, the reversible $\mathrm{RBC}$ aggregation phenomenon dominates. RBC roleaux includes not only the actual volume of the individual globule itself but also the volume of plasma immobilised within the closed aggregate. The convergence to a Newtonian behaviour at high shear rates indicates the predominance of the oriented, deformed and disaggregated hematies in the direction of the flow ${ }^{7}$.

Figure 2 a show a 3-D representation of a human red blood cell. The dimensions of human RBC and porcine RBC are similar. The sequence of micrographies (Fig. 2b to 2e) are from porcine blood at low volume fraction of hematies (hematocrit $\sim 10$ ) diluted in its own plasma and settle be-

Table 1. General data information, maximum and minimum viscosity values measured using a cone-plate type viscometer (see text).

\begin{tabular}{cccc}
\hline number of experiments with: porcine blood $=21$; bovine $=6$; total $=27$ & & & \\
\hline Hematocrit native: porcine: $\max =51$; $\min =40 ;$ & Porcine blood & Bovine blood \\
Maximum and minimum viscosity value & 4.2 & 3.9 & native \\
measured at $\gamma^{\prime}(\mathrm{d} \gamma / \mathrm{dt})=525 \mathrm{~s}^{-1}$ (mPa.s) & 1.9 & 3.0 & 3.6 \\
Maximum and minimum viscosity value & 8.6 & 13.1 & 5.7 \\
measured at $\gamma^{\prime}=11.3 \mathrm{~s}^{-1}$ (mPa.s) & 4.7 & 4.9 & 3.9 \\
\hline
\end{tabular}

Plasma viscosity measured at $\gamma^{\prime}=900 \mathrm{~s}^{-1}$ : porcine blood $=1.7 \mathrm{mPa} . \mathrm{s}$ 
tween two glass slides. Figure $2 b$ show a group of RBC aggregates interconnected. At low shear rate ( low blood velocity) or at "stasis", these aggregates become more and more compact because contact areas increase between them. Fibrinogen macromolecule has often been considered as the most important protein inducing RBC aggregation and a departure from Newtonian behaviour. An elevated level of fibrinogen ( in human beings, normal to pathological range 3 to $7 \mathrm{~g} / \mathrm{l}$ ) is regarded as a risk factor for coronary, cerebral and peripheral vascular disease ${ }^{8}$. In hemorheology it is
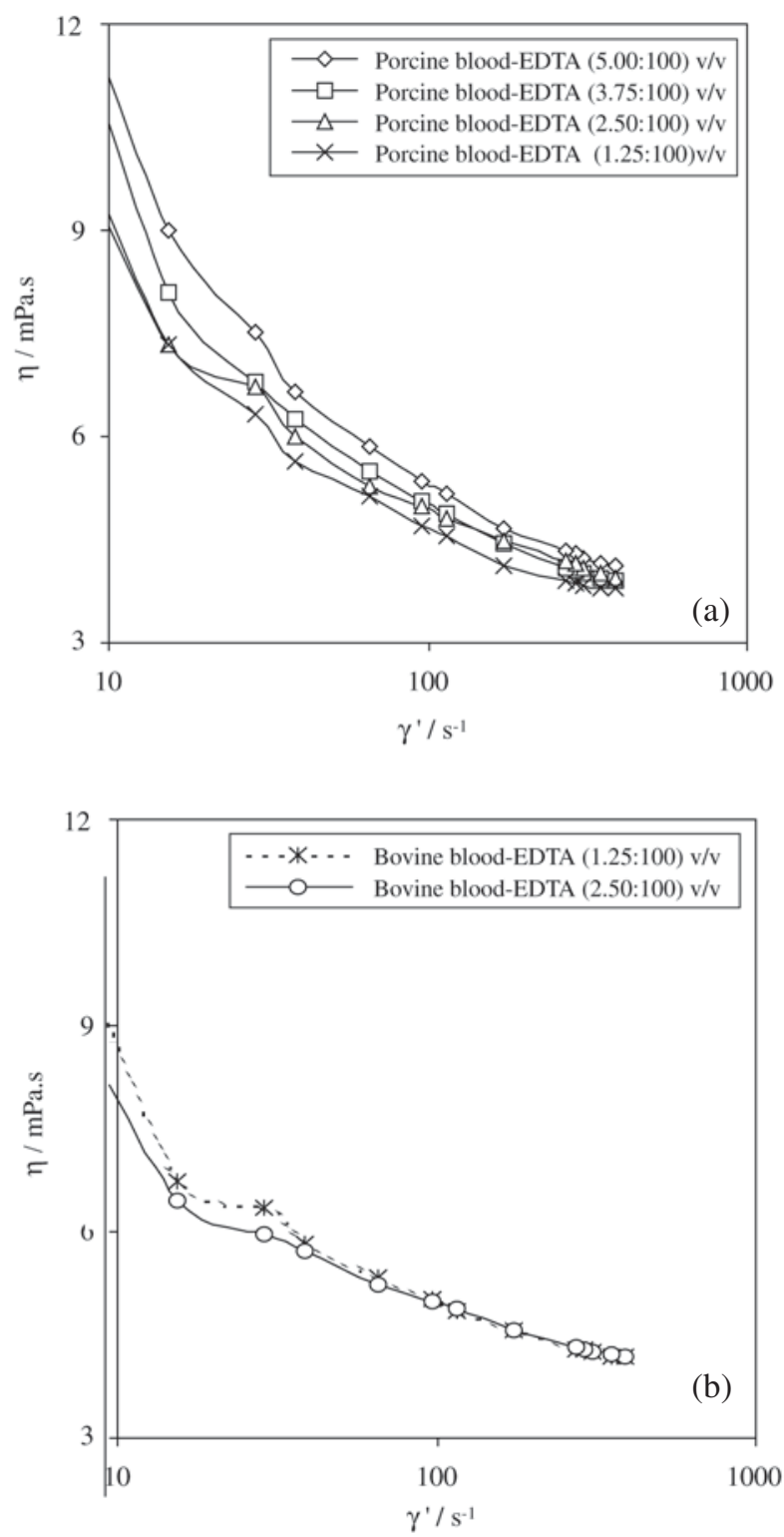

Figure 1. (a) Porcine blood viscosity data at hematocrit $=40$ and increasing EDTA concentration; (b) bovine blood viscosity data at native hematocrit and increasing EDTA concentration. mainly associated with plasma viscosity and RBC aggregation increase and consequently increase in blood viscosity at low shear rates. In human blood fibrinogen macromolecule induce loosely packed network aggregates with large amount of intra-aggregate liquid plasma space. The difference between loosely packed and compact is that the latter has a large inter-aggregate liquid plasma space.

Figure 2c show how deformable a normal porcine red blood cell can be while Figs. $2 \mathrm{~d}$ to $2 \mathrm{e}$ show how this deformability is essential to produce three-dimensional structures. The condition for equilibrium is that three surface tensions should form a triangle of forces. Thus from Fig. 2f, which is a representation of a stable red cell node (B), the arrangement necessary to three-dimensional aggregate growth is

$$
\phi_{\alpha} / \sin \alpha=\phi_{\beta} / \sin \beta=\phi_{\theta} / \sin \theta
$$

Where $\alpha, \beta$ and $\theta$ are the interfacial angles, $\phi_{\alpha}, \phi_{\beta}$ and $\phi_{\theta}$ are the surface tensions, $\mathrm{A}_{1}, \mathrm{~A}_{2}, \mathrm{~A}_{3}$ are three hematies adhered to the central red cell $\mathrm{B}$. The dihedral angle or contact angle $\theta$ adjusts itself to bring the forces into balance, giving

$$
F_{1}=F_{2} \cos (\theta / 2)+F_{3} \cos (\theta / 2)=2 F_{2} \cos (\theta / 2)
$$

In equilibrium, the forces $\mathrm{F}_{2}=\mathrm{F}_{3}, \theta \sim 60^{\circ}$ or lower and $\alpha=\beta \sim 150^{\circ}$ or higher. The equilibrium therefore can receive an approach similar to the one proposed by D'Arcy Thompson ${ }^{9}$ and C.S. Smith ${ }^{10}$ for the movement of boundaries and bubble equilibrium.

This stable RBC node for three-dimensional aggregate growth is marked as number 1 in Fig. 2 d. Like the porcine $\mathrm{RBC}$, the human RBC membrane skeleton is a highly deformable network of spectrin molecules linked together by actin filaments and spectrin self-association interactions. The thickness of the cell can be more than halved as shear stress increases.

At low shear rates or stasis (as it is the case of the present experiment, e.g., blood confined between glass slides) the RBC deformability play a key role on aggregate building. When the local situation do not allow the organisation of the red cell node the equilibrium is attained via the change in shape (spheroidisation) of the cell as marked as 2 in Fig. 2d.

Aggregate compaction occurs after they became closed structures. Viewed from the centre of a closed RBC aggregate, the concave boundaries (marked as number 3 in Fig. 2b) will tend to move towards the centre of the intraspace and therefore eliminating intra-aggregate plasma space. The consequence is the whole aggregate becoming compact. The side-to-side attachment is a consequence of this process of closing intra-aggregate space ${ }^{11}$. The consequence of compaction is the expulsion of plasma and the increase in aggregate density. The aggregate nodes will also 
force a process of inter-locking between the different parts of the aggregates. Higher density and interlocking will favour aggregate sedimentation.

It is known that red cell deformability is dependent on the red cell surface area to volume ratio $(\mathrm{A} / \mathrm{V})$, the internal viscosity of the red cell, the membrane viscoelasticity, mem- brane surface electrical charges, the volume fraction (hematocrit) and shear stress. Low A/V or small red cell diameter could result in membrane bending rigidity and therefore a low level of erythrocyte aggregation. Considerable animal species variations in the red cell $\mathrm{A} / \mathrm{V}$ parameter, mean cell volume (MCV) and diameter has been re-
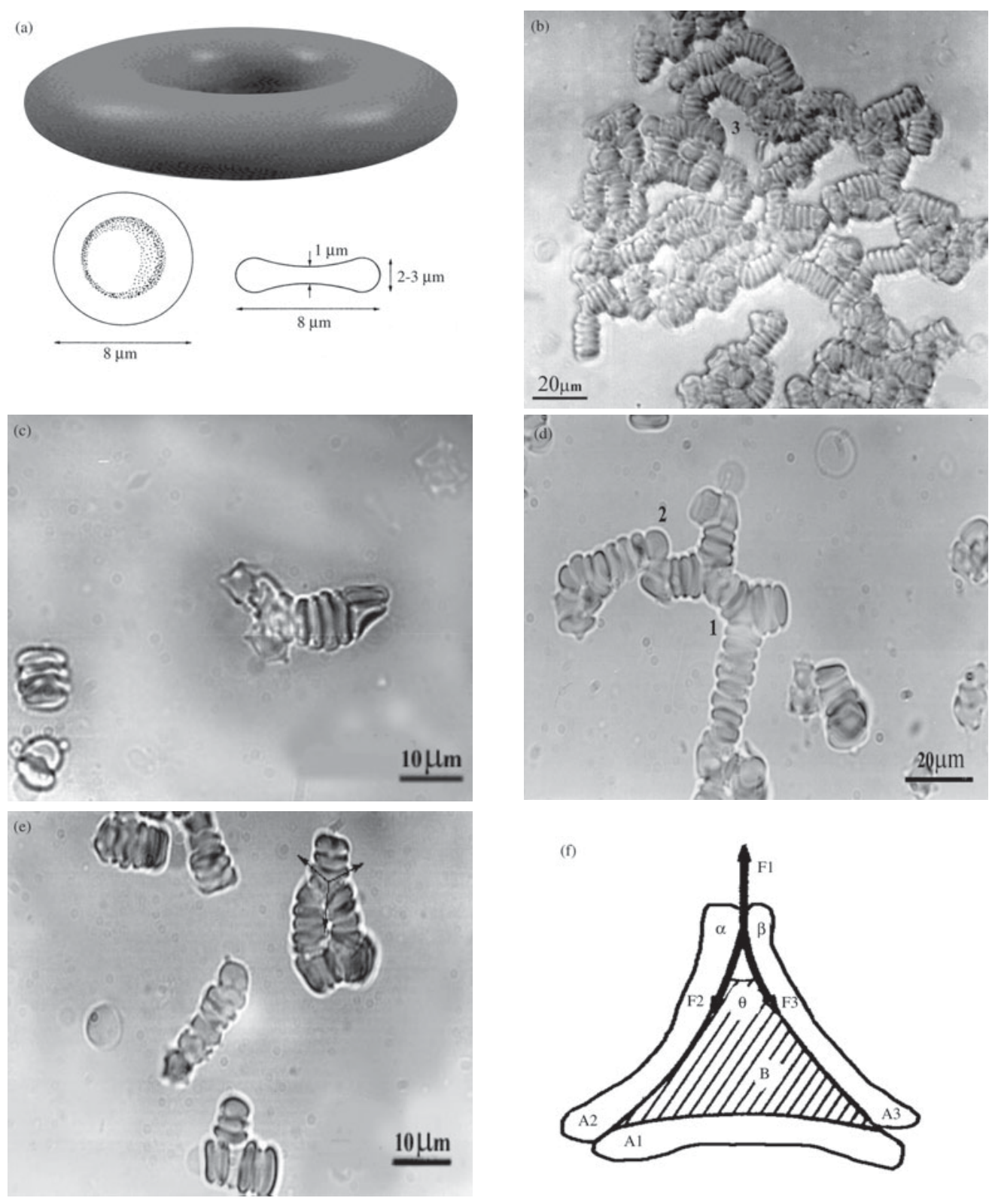

Figure 2. (a) 3-D representation and dimensions of human red blood cells; Porcine blood: (b) Optical photomicrograph of closed red blood cell aggregates. Intra-space aggregate tend to decrease by the movement of the concave sides toward the centre of the aggregate (3); (c) Optical photomicrograph of a deformed red blood cell; (d) Optical photomicrograph red blood cell stable node deformed as a triedro (1) or sphere (2); e) Optical photomicrograph of the a red cell node(deformed to a triedro shape ); f) Representation of a stable red blood cell node. $\mathrm{A}_{1}, \mathrm{~A}_{2}, \mathrm{~A}_{3}$ are three hematies adhered to the deformed central red blood cell $\mathrm{B}$. 
ported since the pioneering work of W. Hewson (1774) ${ }^{1}$. These differences may contribute to the animal species differences in erythrocyte aggregation.

Figure 3 show optical micrographs of experiments using bovine blood samples. Contrary to human and porcine blood, bovine blood show little or no aggregation phenom- enon as depicted in Fig. 3a. To study the interaction of nonaggregate red blood cells with air bubble surfaces we have intentionally produced air bubbles between glass slides. As shown in Fig. 3b, there is no aggregation of bovine cells at the bubble surface. The bovine RBC slides each other as well as the bubble surface and no attachment is observed
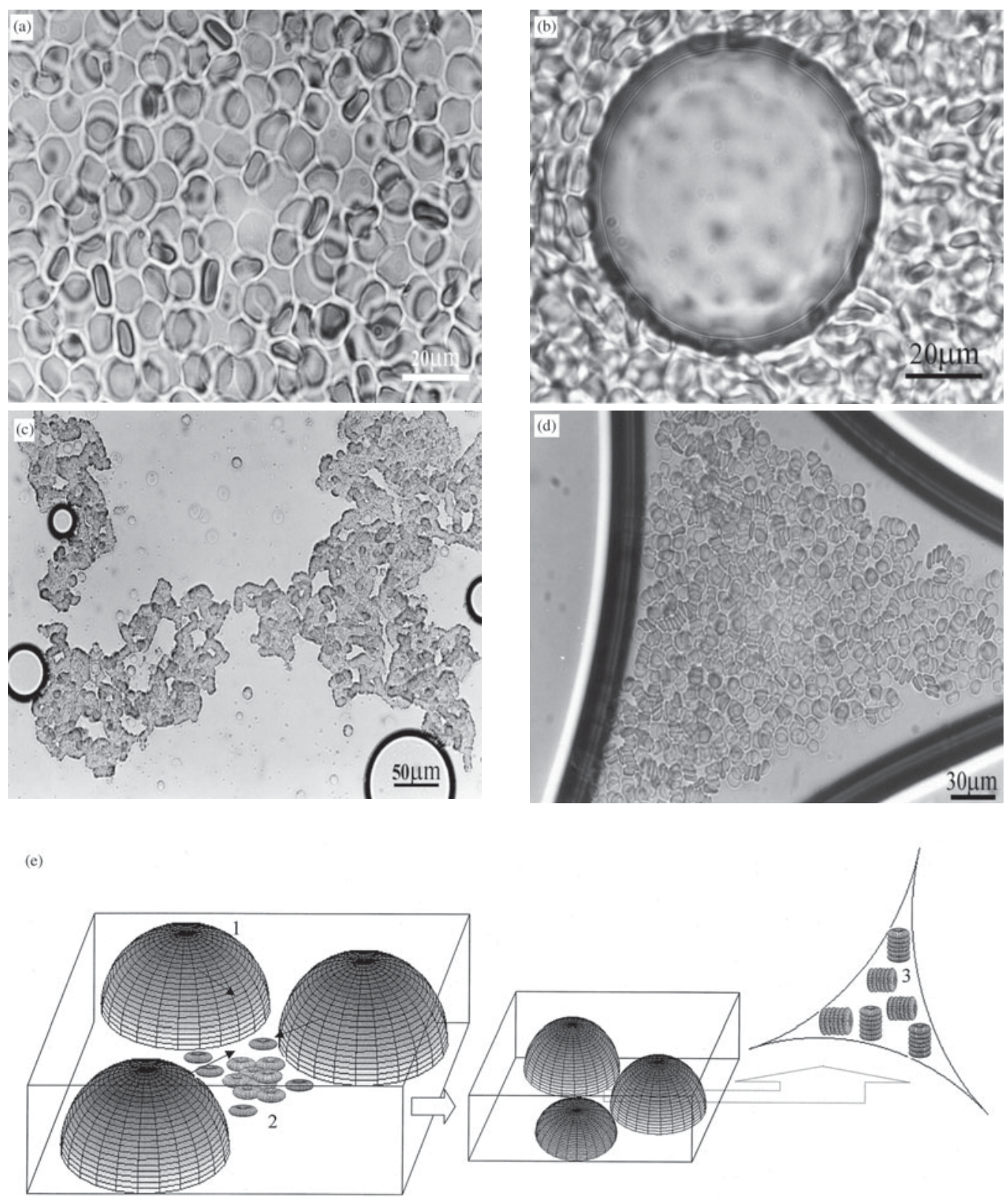

Figure 3. Bovine blood: (a) Optical photomicrograph of bovine red blood cells; (b) Bovine red blood cells do not attach to the bubble; (c) Porcine red blood cells attached to the bubbles; (d) Optical photomicrograph of blood entrapped between three air bubbles (see Fig. 10e): under pressure natural non-aggregate bovine blood will tend to organise in roleaux; (e) Experiment under optical microscope (sketch, see photomicrograph 3d): 1- Air bubble between two glass slides; 2- bovine red cell; 3- Bovine RBC forming roleaux under pressure. 
between them. This behaviour is opposite to porcine RBC interaction with air bubbles as depicted in Fig. 3c. Here the porcine red cell aggregates are attached to all the air bubbles.

To analyse the behaviour of bovine RBC cells we have performed the experiment illustrated in Fig. 3e. There three air bubbles entrapped an amount of bovine RBC in their own plasma. Due to the static pressure (not quantified) exerted by the three bubbles arrangement the bovine RBC's tend to organise in roleaux (micrograph shown in Fig. 3d) probably to reduce the pressure gradient via minimising free surface area. Increasing local pressure can therefore enhance aggregation from normally non-aggregating red blood cells.

\section{Conclusions}

Using porcine blood samples (whose rheology is similar to human blood) it is was possible to visualise how linear arrays of RBC's (roleaux) at low shear rates or stasis, turned to three-dimensional aggregates via the occurrence of stable dihedral angles between adjacent red cells. At very low shear rate or stasis, the process of aggregate building in the porcine blood sample can be summarised as follow:

Uni-dimensional RBC roleaux $\rightarrow$ formation of nodes between roleaux $\rightarrow$ three-dimensional growth $\rightarrow$ aggregate closing by end-to-end attachment.

When the node assumes triedro geometry the aggregate become stable and three-dimensional growth could be started. Geometrical aspects are crucial on the compaction of red blood cells. The plasma intra-space aggregates tend to decrease in area and eventually disappear as the concave sides move towards the direction centre of the plasma intraspace. Consequently intra-spaces decrease and red blood cell aggregates increase in compaction and density. This could eventually lead to the formation of dense shear-resistant RBC clusters, depending on how the equilibrium $\mathrm{RBC}$ nods and the movement of concave sides of plasma intra-space aggregates produce interlocking structures with higher internal pressure. These clusters depending on their location and local blood flow velocity might eventually sediment.

Finally, the microstructure study of normally non-aggregate blood samples such as bovine blood could help un- derstanding the role of increasing local pressure on the microrheology of red blood cells. Under pressure it seem that bovine blood sample show roleaux formation probably to reduce surface area and consequently pressure gradient.

\section{Acknowledgements}

The authors are indebted to STQ-CETEC for financial support and to Profs. Renaldo Travassos Martins, Israel José da Silva(School of Veterinary Medicine-UFMG) and $\mathrm{Mr}$ Antonio José (SECAR/DIPOA-MAA) for their invaluable help.

\section{References}

1. Bitbol, M. "Chapitre I: Histoire de l'hémorheologie”,Doctarate Thesis, University Paris VII, Paris, France, 1985.

2. Chien, S.; Sung, L.A. $1^{e r}$ Symp. Int. Hémorhéologie et agrégation érythrocytaire, ed. Médicales Internationales, v. 1, p. 122-147, Paris, 1986.

3. Quemada, D. Eur. Phys. J. App., v. 1, p. 119-127, 1998.

4. Weng, X.; Cloutier, G.; Pibarot, P.; Durand, L.-G.; Biorheology, v. 33, n. 4,5, p. 365-377, 1996.

5. Snabre, P.; Haider, L.; Mills, P. in Proc. "Rheologie des materiaux du vivant" $-34^{\text {th }}$ Annual Meeting of the French Soc. of Rheology, p. 20-29, 1999.

6. Agosti, R.; Clivati, A.; D’Ettorre, M.; Ferrarini, F.; Somazzi, R.; Longhini, E. Clinical Hemorheology, v. 8, p. 913-924, 1988.

7. Quemada, D.; Dufaux, J.; Flaud, P. La Recherche, v. 24, p. 584-590, 1993.

8. Levenson, J. in The Expert Speak 1997, ed. by K. Simpson, J. Ekdahl, K. R. Hamilton, interview.p. 38-39, Sacramento, Ca, 1997.

9. Thompson, D'Arcy W. On growth and form, Cambridge Univ. Press, v. II, $2^{\text {nd }}$ ed , p. 465-472, Cambridge, rep. 1953.

10. Cottrell, A. An introduction to metallurgy, E. Arnold, London, p. 340-344, rep 1985.

11. Hansen, J.C.; Skalak, R.; Chien, S.; Hoger, A. Biorheology, v. 34, n. 4/5, p. 327-348, 1997.

12. Schmid-Schönbein, H.; Malotta, H.; Striesow, F. Tijdschr NVKC, p. 88-97, 1990. 